\title{
Constraining GRB progenitor models by probing Wolf-Rayet wind geometries in the Large Magellanic Cloud
}

\author{
Jorick S. Vink ${ }^{1,2}$ \\ 1 Keele University, Lennard Jones Laboratories, Keele, Staffordshire ST5 5BG, UK \\ 2 Armagh Observatory, College Hill, Armagh BT61 9DG, Northern Ireland, UK \\ e-mail: jsv@arm.ac.uk
}

Received 14 February 2007 / Accepted 8 April 2007

ABSTRACT

\begin{abstract}
Context. The favoured progenitors of long-duration gamma-ray bursts (GRBs) are rapidly rotating Wolf-Rayet (WR) stars. However, most Galactic WR stars are slow rotators, as stellar winds are thought to remove angular momentum. This poses a serious challenge to the collapsar model. Recent observations indicate that GRBs occur predominately in low metallicity $(Z)$ environments, which may resolve the problem: lower $Z$ leads to less mass loss, which may inhibit angular momentum removal, allowing WR stars to remain rotating rapidly until collapse.

Aims. We wish to determine whether low $Z$ WR stars rotate on average more rapidly than Galactic WR stars.

Methods. We perform a Very Large Telescope (VLT) linear spectropolarimetry survey of WR stars in the low $Z$ environment of the Large Magellanic Cloud (LMC) and compare our results with the Galactic sample of Harries et al. (1998, MNRAS, 296, 1072).

Results. We find that only 2 out of 13 (i.e. 15\%) of LMC WR stars show line polarization effects, compared to a similarly low fraction of $\sim 15-20 \%$ for Galactic WR stars.

Conclusions. The low incidence of line polarization effects in LMC WR stars suggests that the threshold metallicity where significant differences in WR rotational properties occur is below that of the $\mathrm{LMC}\left(Z \sim 0.5 Z_{\odot}\right)$, possibly constraining GRB progenitor channels to this upper metallicity.
\end{abstract}

Key words. stars: Wolf-Rayet - stars: early-type - techniques: polarimetric - gamma rays: bursts

\section{Introduction}

A picture is emerging in which long-duration gamma-ray bursts (GRBs) are associated with the endpoints of the life of a massive star (Galama et al. 1998; Hjorth et al. 2003; Stanek et al. 2003). This has given huge impetus to the collapsar model for gamma-ray bursts of Woosley (1993). The model relies on both the progenitor being rapidly rotating, allowing it to subsequently collapse to a black hole, as well as the progenitor being a relatively compact star that has lost its hydrogen $(\mathrm{H})$ envelope, allowing the jet to emerge. The progenitor is therefore generally considered to be a H-poor Wolf-Rayet (WR) star (e.g. Mirabal et al. 2003).

The challenge of these two requirements is that they seem mutually exclusive. Massive stars posses strong stellar winds, which may remove the angular momentum (Langer 1998; Maeder \& Meynet 2000), leaving a slowly rotating WR star before collapse.

However, the winds of massive stars are predicted to scale with the metallicity (Abbott et al. 1982; Kudritzki et al. 1987; Leitherer et al. 1992; Vink et al. 2001), which would imply that stars in lower metallicity environments could rotate faster. A very relevant development in the predictions of the metallicity dependence of stellar winds has been that WR mass loss should scale with the Fe content of the host galaxy (Vink \& de Koter 2005; Gräfener \& Hamann, in prep.), and not with self-enriched species - as previously often assumed (e.g. Maeder \& Meynet 2003). The steep scaling of WR mass loss with $\dot{M}$ proportional to $Z^{0.6-0.8}$, as predicted by Vink \& de Koter (2005) appears to be confirmed by observations (Crowther 2006). The metallicity dependence of WR stars is also backed up with stellar evolution models that can account for the drop in WC/WN ratio in galaxies at low $Z$ (Eldridge \& Vink 2006; see also Van Bever \& Vanbeveren 2003).

Another intriguing aspect is that the inferred steepness of the WR mass loss $Z$ dependence may explain the inhibition of angular momentum removal from WR stars at low $Z$, presenting a boost to the collapsar model for GRBs at low metal content (Yoon \& Langer 2005; Woosley \& Heger 2006). Therefore, the earlier mentioned paradox for the explanation of long-duration GRBs from rapidly rotating WR stars may be resolved if they would occur at low $Z$. Intriguingly, recent observations indeed indicate that GRBs are favoured in regions of low metallicity (e.g. Fynbo et al. 2003; Prochaska et al. 2004; Vreeswijk et al. 2004).

In this paper, we test the physical criteria of GRB progenitors observationally. If the WR mass-loss metallicity dependence and the subsequent inhibition of angular momentum removal are indeed the key to account for the predominant occurrence of GRBs at low Z, WR stars in the Magellanic Clouds should be rotating more rapidly than those in the Galaxy. We therefore wish to infer the fraction of rapidly rotating WR stars in the low-Z environment of the LMC and compare it to that in the Galaxy. Unfortunately, direct $v \sin i$ measurements are not feasible for WR stars but rapid rotation is believed to induce wind asymmetries (e.g. Maeder \& Meynet 2007) which can be probed with linear spectropolarimetry. 
Despite this tool being "photon-hungry", linear spectropolarimetry is a powerful technique to measure asymmetries, as one of the basic principles is the following: continuum light is polarized by Thomson scattering off free electrons. For the case of a spatially unresolved spherical wind, polarized photons from all directions cancel, leaving no net polarization signal. In case the wind departs from spherical symmetry, a net continuum polarization should be detected. Line photons are formed by recombinations over a larger volume and scatter and polarize less than the continuum photons. As a result, the line becomes depolarized with respect to the continuum - if the wind is asymmetric.

The method has been extensively used for rapidly rotating Be stars. Poeckert \& Marlborough (1978) found $~ 60 \%$ of Be stars to be intrinsically polarized, which is, due to random orientation of rotational axes, consistent with all Be stars having asymmetric outflows induced by rapid rotation. In a similar vein, Harries et al. (1998) performed a linear spectropolarimetry survey on 16 Galactic WR stars and found line effects in only $15-20 \%$ of them. Their results suggested that only a small fraction of Galactic WR stars have significant asymmetric outflows due to rapid rotation.

Here, we present a Very Large Telescope (VLT) linear spectropolarimetry survey on a complete sample of the brightest LMC WR stars (with $V \lesssim 12.3$ ) and we compare our results to the Galactic sample. If WR stars at low $Z$ indeed remain rapidly rotating, we may measure a higher fraction of line depolarizations.

\section{Observations}

Our targets were selected from the fourth catalogue of Population I WR stars in the LMC by Breysacher et al. (1999; hereafter BAT) on the basis of their relative brightness $(V \lesssim$ 12.3). Our LMC sample-size (of 13) is comparable to that from the Galactic WR spectropolarimetry study by Harries et al. (1998). We note that targets were not chosen on the basis of any known circumstellar geometries. As we currently know little about the polarimetric evolution of WN (nitrogen rich) into WC (carbon rich) into WO (oxygen rich) stars, nor do we know which of these subgroup WR stars may give rise to GRBs, we first wish to establish the overall difference in rotationally induced wind asymmetry between the LMC and the Galaxy. The list of objects is given in Table 1, alongside their $V$ magnitudes and spectral types.

The linear spectropolarimetry data were obtained during the nights of 2006 October 29 and 30 using the FORS 1 spectrograph on the VLT UT2 (Kuyen) in PMOS mode. The exposure times are given in Col. (4) of Table 1. The observations were obtained in a very similar manner to that of the Herbig Ae/Be study by Vink et al. (2002) to which we refer for details.

We used the GRIS600R+14 grism (and the GG435+31 order filter) with a slit width of $0.51^{\prime \prime}$, which yielded a resolution of approximately $3 \AA$, i.e. $150 \mathrm{~km} \mathrm{~s}^{-1}$ around $6500 \AA$, i.e. a very similar setting to the Galactic WHT study by Harries et al. (1998). Due to relatively poor seeing we increased the slit width to $0.8^{\prime \prime}$ during parts of the run. Depolarizations can be measured across the He II line at $6560 \AA$ and several other emission lines, such as the He II line at $5976 \AA$ and the CIV line at $5801 \AA$ for WC stars. As WR stars have fast winds (of order $\sim 1500-5000 \mathrm{~km} \mathrm{~s}^{-1}$ ), we are able to resolve these lines.

To analyze the linearly polarized component in the spectra, FORS1 was equipped with the appropriate polarization optics. Polarization and un-polarized standard stars were also observed.
Table 1. WR targets. The $V$-band magnitudes are taken from from Breysacher et al. (1999, BAT) and listed in Col. (2). The Spectral types (Col. 3) are taken from BAT (see references therein). The integration times (Col. 4) denote the total on source exposures. The observed continuum position angle $(\Theta)$ and its error are measured between $5900 \AA$ and $6500 \AA$ and given in Cols. (5) and (6).

\begin{tabular}{lclrcc}
\hline \hline BAT & $V$ & Spec & $\operatorname{Exp}(\mathrm{s})$ & $P_{\text {cont }}(\%)$ & $\Theta_{\text {cont }}\left({ }^{\circ}\right)$ \\
\hline 22 & 12.09 & WN9h & 1600 & $0.235 \pm 0.007$ & $147.8 \pm 0.9$ \\
33 & 11.54 & WN9 & 1400 & $1.315 \pm 0.007$ & $106.2 \pm 0.2$ \\
\hline 27 & 11.31 & WN5?b & 1400 & $0.254 \pm 0.006$ & $36.3 \pm 0.7$ \\
28 & 12.23 & WC6 & 2400 & $1.038 \pm 0.007$ & $47.5 \pm 0.2$ \\
38 & 11.50 & WC4 & 1600 & $0.556 \pm 0.007$ & $21.1 \pm 0.3$ \\
39 & 12.51 & WC4 & 1000 & $0.445 \pm 0.013$ & $23.0 \pm 0.9$ \\
42 & 9.91 & WN5?b & 360 & $0.601 \pm 0.007$ & $24.5 \pm 0.4$ \\
55 & 11.99 & WN11h & 1200 & $0.226 \pm 0.009$ & $30.7 \pm 1.1$ \\
85 & 11.75 & WC4 & 1600 & $1.716 \pm 0.007$ & $104.1 \pm 0.1$ \\
92 & 11.51 & WN6 & 1200 & $1.043 \pm 0.006$ & $81.6 \pm 0.2$ \\
107 & 12.12 & WNL & 2400 & $1.647 \pm 0.006$ & $70.1 \pm 0.1$ \\
118 & 11.15 & WN6h & 1200 & $0.166 \pm 0.006$ & $20.6 \pm 1.1$ \\
119 & 12.16 & WN6(h) & 2400 & $2.231 \pm 0.006$ & $81.2 \pm 0.1$ \\
\hline
\end{tabular}

The data reduction was carried out using IRAF for the usual bias and sky-subtraction, cosmic ray removal, spectrum extraction and wavelength calibrations of the (extra)ordinary rays, and STARLINK software to determine $Q$ and $U$, as well as the percentage linear polarization $P$ and its position angle $\Theta$. The achieved accuracy of the polarization data is in principle determined by photon-statistics only, which can be rather good $(<0.01 \%$; see Table 1). However due to systematic effects, the absolute accuracy is lower. We do not correct for interstellar polarization (ISP) as these are equal for line and continuum wavelengths, but we note that interstellar polarization may act in a way that depolarization of the source turns into an enhancement of $P$ (and an accompanying change in $\Theta$ ) in the data (see e.g. BAT 22 below).

\section{Results}

The continuum polarization percentage and its position angle are measured over a region between $5900 \AA$ and $6500 \AA$ and given in Cols. (5) and (6) of Table 1. Figures 1 and 2 show the polarization data of all targets in the form of triplots. The lower panels show the Stokes I "intensity" data, the linear polarization percentages are plotted in the middle panels and the position angles are in the upper triplot panels. The polarization data are binned to a constant error of $0.05 \%$.

The only two objects that appear to be subject to significant intrinsic polarization (with $\gtrsim 0.3 \%$ ) are BAT 22 and BAT 33 for which line polarizations effects can be observed across the He II line (at $16560 \AA$ ) as well as at least one other line. For, BAT 118 we cannot definitively exclude a line effect, but because it would at most represent a marginal detection, we count it amongst the non-detections and include it in Fig. 2.

The clearest detection is that of BAT 33. Depolarization can be observed in the middle panel of the left-hand side of Fig. 1 across both the He II lines at $6560 \AA$ and at $5976 \AA$, whilst the position angle remains constant across the lines. The case of BAT 22, also designated R 84, is less straightforward. Here, the polarization increases across the He II $6560 \AA$ line, whilst the PA is also found to change. This may be due to an alignment of the interstellar and intrinsic polarization (see e.g. Vink et al. 2005, for the extreme case of FU Ori). We can estimate the maximum amount of interstellar foreground polarization using the standard relation between $A_{\mathrm{V}}$ and $\% P, \frac{P^{\max }}{A_{\mathrm{V}}}=3$ of 

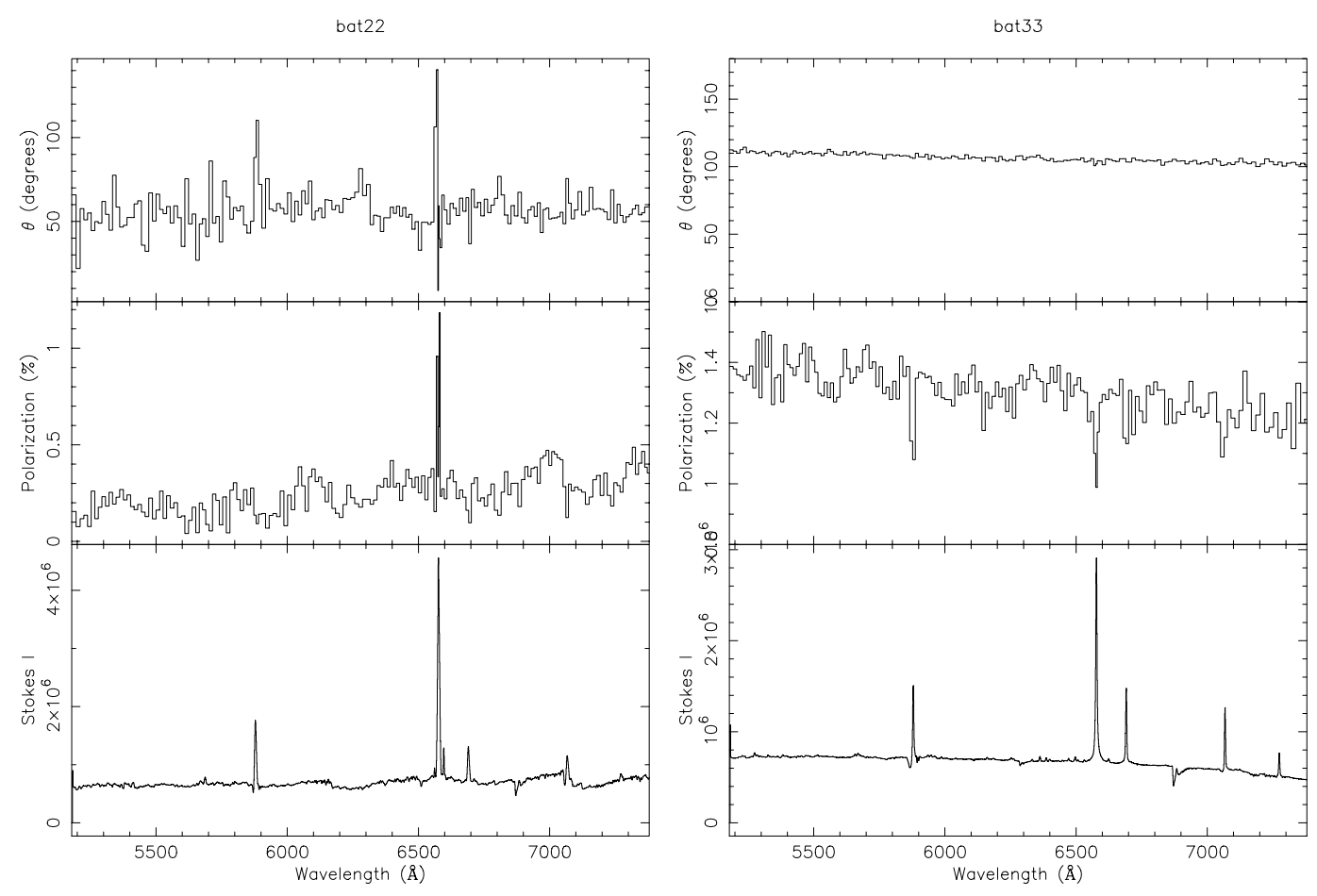

Fig. 1. Polarization triplots for the WR targets that show depolarization. The Stokes $I$ spectra are shown in the lower panel, the \%Pol are shown in the middle panel, and the position angles are plotted in the upper panel of the triplots.

Serkowski et al. (1975). Heydari-Malayeri et al. (1997) derive a relatively large $A_{\mathrm{V}}$ of 0.75 , which could result in a maximum linear polarization of $\sim 2 \%$ using the relation between ISP and reddening. We note that these values are maximal and highly uncertain (due to the uncertainties in the used Serkowski relation). In addition, we cannot exclude the possibility that the line itself is polarized. Line polarization may vary for lines formed at different positions in the wind, which may result in differing behaviour of the He II $6560 \AA$ and $5976 \AA$ lines. A further discussion on $\mathrm{R} 84$, often termed a "transitional object" would require more in-depth modeling, which is beyond the scope of this paper.

Harries et al. (1998) found the Galactic line-object WR stars to be amongst the brightest, largest mass-loss rate systems and we now examine whether the LMC line-effect objects are also "special" in terms of their stellar parameters. Four (out of 13) of our LMC objects, BAT 18, BAT 55, BAT 118 and BAT 119, have been analyzed by Crowther \& Smith (1997) with the aim of determining the photospheric/wind parameters. Although BAT 22 and BAT 118 are indeed high luminosity/large mass-loss systems, so is BAT 119, for which we have not detected a line effect. The current sample is too small to assess whether wind asymmetry of LMC WR stars is restricted to large mass-loss systems.

Regarding duplicity, Foellmi et al. (2003) recently performed a detailed investigation into the multiplicity of LMC WR stars. Unfortunately, their sample, and our brighter sample, have no objects in common. At this stage, it is thus not possible to assess whether the line-effect systems are binaries and whether the polarization effects might be due to asymmetric electron scattering around rapidly rotating single stars, or whether the polarization might be the result of intra-binary scattering instead.

All in all, we put the incidence of line effects at $2( \pm 1)$ out of a sample of 13 LMC WR objects, corresponding to a line effect frequency of $\sim 15 \%$. Equation (23) of Brown \& McLean (1977) shows that the percentage polarization scales as
$P \propto \sin ^{2} i$, implying that we may not detect any polarization for low-inclination objects - even if their winds are significantly axisymmetric. Our data are of similar quality (in terms of $\mathrm{S} / \mathrm{N}$ ) as those in Harries et al. (1998) and the incidence of line effects is comparably low to that of the $\sim 15-20 \%$ of line effects detected in Galactic WR stars by Harries et al.

\section{Discussion and conclusions}

In terms of their polarization properties, the LMC WR stars appear to be indistinguishable from their Galactic counterparts, and one may wonder whether WR properties are simply the same at different metallicities. Recently, Marchenko et al. (2007) performed a spectroscopic monitoring campaign of Small Magellanic Cloud (SMC) WR stars and found their moving subpeaks to behave similarly in the SMC as in the Galaxy. Rather than due to global rotation-induced asymmetric structures, one may wonder if the WR polarization may also be due to wind clumping, as is believed to be the case for Luminous Blue Variables (Davies et al. 2005; see also Nordsieck et al. 2001). However, Harries et al. (1998) considered this option of inhomogeneous winds to account for the Galactic WR distribution of polarization levels, but found the statistically most significant results when a 15-20\% minority of WR stars reached measurable depolarizations due to flattened winds, whilst the large majority were slow rotators with spherically symmetric winds. The conclusion was drawn that the inferred asymmetries were only present for the most rapidly rotating WR stars (see also Villar-Sbaffi et al. 2006).

Taking our results and those of Harries et al. (1998) at face value, we may conclude that there is no significant difference in the rotational properties of WR stars in the LMC and the Galaxy, unless the physical requirements to produce polarization are significantly more stringent for WR stars at LMC metallicity. However, intrinsic linear polarization is observed for WR stars with a range of mass-loss rates and metallicities, as well as for 
bot 27

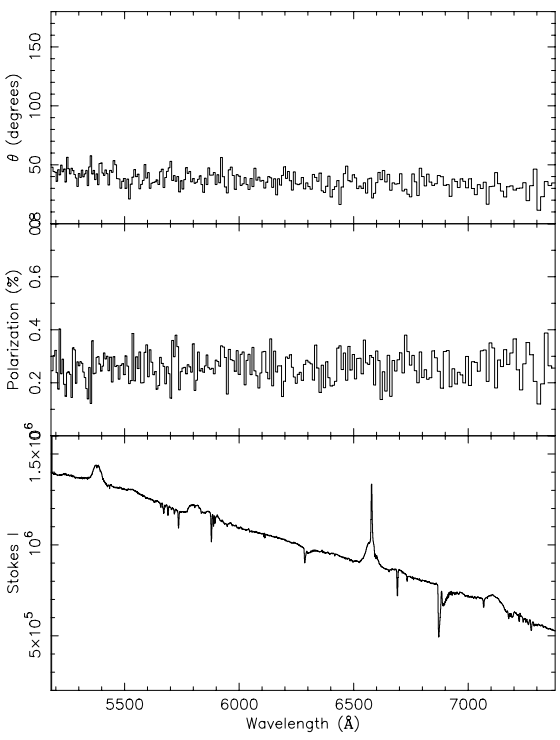

bat39

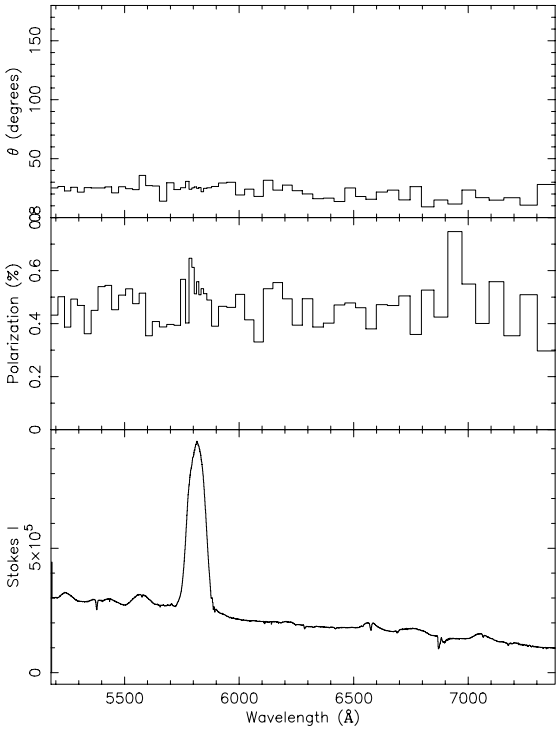

bat8

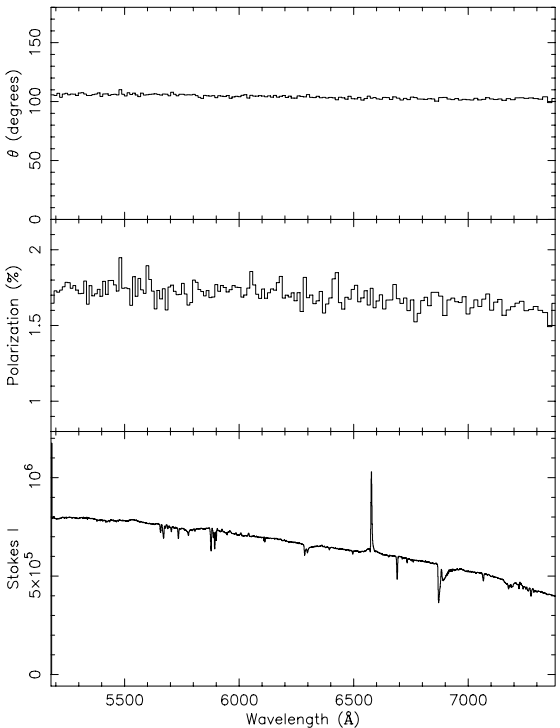

$5+28$
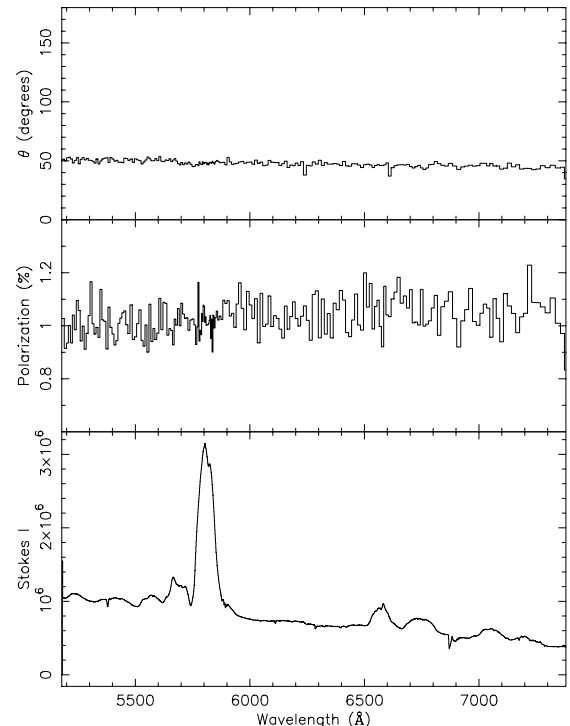

oot 42
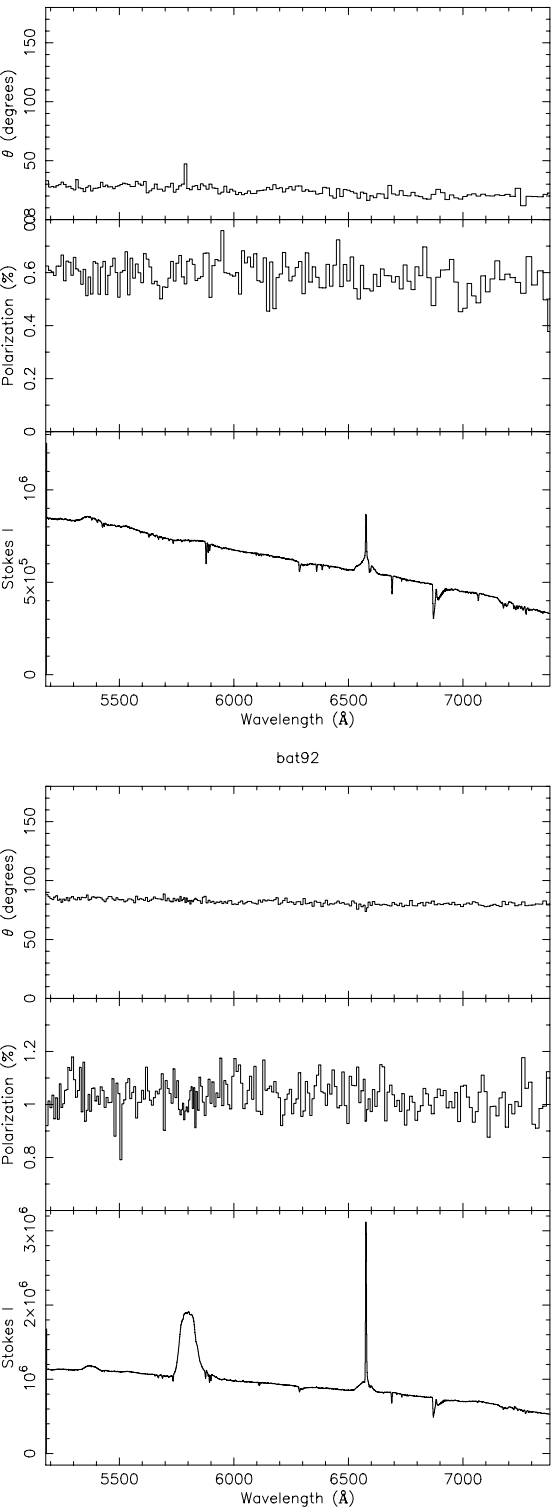

$+38$

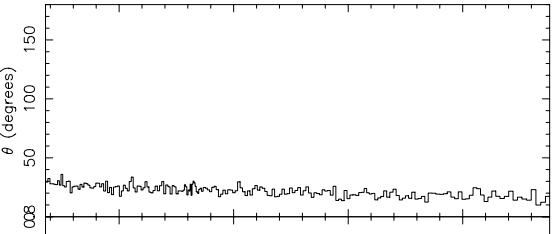

8:

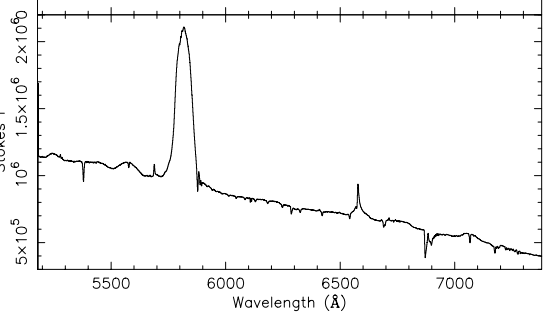

oot55

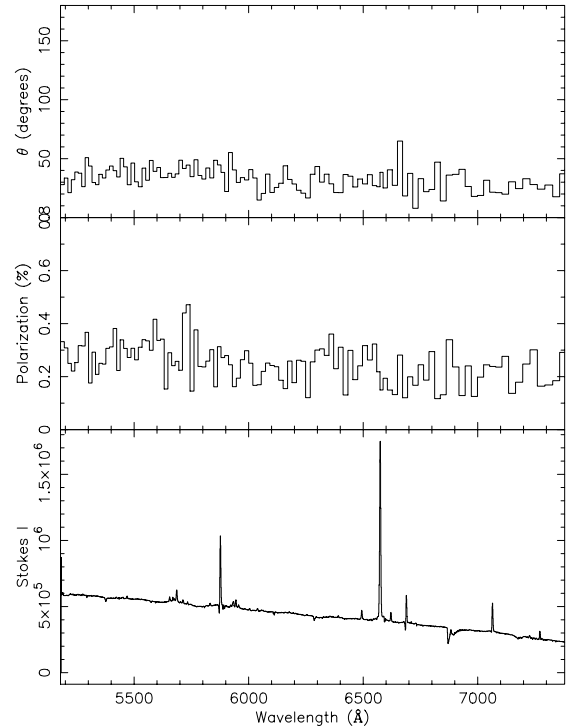

bat107

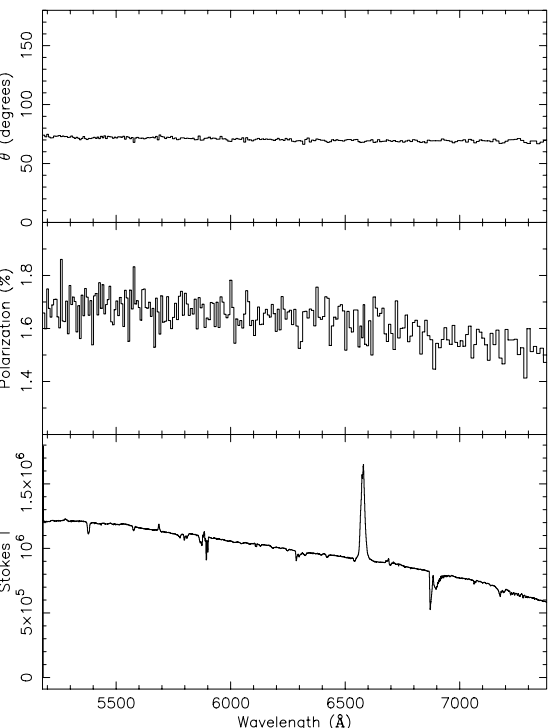

Fig. 2. Polarization triplots for the WR stars that do not show line depolarizations. 

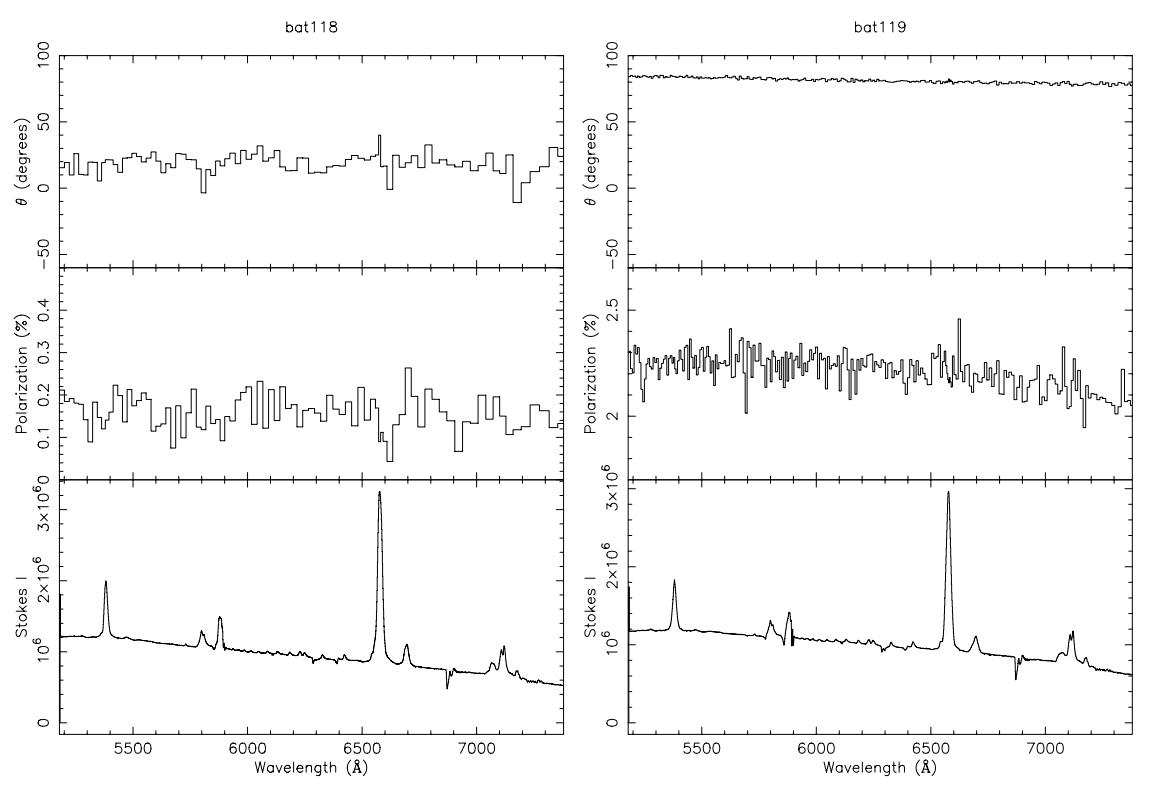

Fig. 2. continued.

objects with much smaller mass-loss rates than WR stars (cf. classical Be stars). It is therefore most likely that the rotational properties of LMC WR stars are indistinguishable from those in the Galaxy, with just a handful of objects rotating rapidly, whilst the majority rotate slowly. In other words, mass loss appears to be sufficient to remove the angular momentum not only in the Galaxy, but also in the LMC.

We speculate that one would need to assess WR stars at lower metallicity than the the $Z \sim 0.5 Z_{\odot}$ of the LMC, for the winds to be weak enough to prevent angular momentum loss. A spectropolarimetric survey in a $Z \sim 0.2 Z_{\odot}$ environment would be extremely useful, however the faintness of SMC WR stars prevents a spectropolarimetric survey of these objects - even with an $8 \mathrm{~m}$-telescope. Future spectropolarimetric programs with Extremely Large Telescopes may address the question of the rotationally induced polarization properties of GRB progenitors more conclusively, if these instruments are equipped with the required polarization optics. In addition, the effect of low $Z$ wind asymmetries on GRB afterglow observations would be interesting (see Eldridge 2007).

Wolf \& Podsiadlowski (2007) recently suggested that GRB progenitor models that require a metallicity much less than $Z \sim 0.5 Z_{\odot}$ appear inconsistent with constraints from hostgalaxy luminosities (however see also Stanek et al. 2006). Here, the low incidence of WR polarization signals suggests that the metallicity threshold where significant differences in WR rotational properties might occur is below that of the LMC. Hence, we may constrain GRB progenitor models to an upper metallicity of that of the $\mathrm{LMC}$, i.e. $Z \sim 0.5 Z_{\odot}$.

Acknowledgements. I would like to thank the referee, Tim Harries, for constructive comments that have helped improve the paper and Rubina Kotak and the friendly staff at ESO/Paranal for their help.

\section{References}

Brown, J. C., \& McLean, I. S. 1977, A\&A 57, 141

Crowther, P. A. 2006, ASPC 353, 157C

Crowther, P. A., \& Smith, L. J. 1997, A\&A 320, 500

Davies, B., Oudmaijer, R. D., \& Vink, J. S. 2005, A\&A, 439, 1107

Eldrige, J. J. 2007, MNRAS, accepted [arXiv: astro-ph/0701707]

Eldridge, J. J., \& Vink, J. S. 2006, A\&A, 452, 295

Foellmi, C., Moffat, A. F. J., \& Guerrero, M. A. 2003, MNRAS, 338, 1025

Fynbo, J. P. U., Jakobsson, P., Möller, P., et al. 2003, A\&A, 406, 63

Galama, T. J., Vreeswijk, P. M., van Paradijs, J., et al. 1998, Nature, 395, 670

Harries, T. J., Hillier, D. J., \& Howarth, I. D. 1998, MNRAS, 296, 1072

Heydari-Malayeri, M., Courbin, F., Rauw, G., Esslinger, O., \& Magain, P. 1997, A\&A, 326, 143

Hjorth, J., Sollerman, J., Moller, P., et al. 2003, Nature, 423, 847

Kudritzki, R.-P., Pauldrach, A., \& Puls, J. 1987, A\&A, 173, 293

Langer, N. 1998, A\&A, 329, 551

Leitherer, C., Robert, C., \& Drissen, L. 1992, ApJ, 401, 596

Maeder, A., \& Meynet, G. 2000, A\&A, 361, 159

Maeder, A., \& Meynet, G. 2003, A\&A, 404, 975

Marchenko, S. V., Foellmi, C., Moffat, A. F. J., et al. 2007, ApJ, 656, L77

Meynet, G., \& Maeder, A. 2007, A\&A, 464L, 11

Mirabal, N., Halpern, J. P., Chornock, R., et al. 2003, ApJ, 595, 935

Nordsieck, K. H., Wisniewski, J., Babler, B., et al. 2001, ASP, 233, 261

Poeckert, R., \& Marlborough, J. M. 1978, ApJS, 38, 229

Prochaska, J. X., Bloom, J. S., Chen, H.-W., et al. 2004, ApJ, 611, 200

Serkowski, K., Mathewson, D. L., \& Foid, V. L. 1975, ApJ, 196, 261

Stanek, K. Z., Matheson, T., Garnavich, P. M., et al. 2003, ApJ, 591, L17

Stanek, K. Z., Gnedin, O. Y., Beacom, J. F., et al. 2006, AcA, 56, 333

Van Bever, J., \& Vanbeveren, D. 2003, A\&A, 400, 63

Villar-Sbaffi, A., St-Louis, N., Moffat, A., \& Piirola, V. 2006, ApJ, 640, 995

Vink, J. S., \& de Koter, A. 2005, A\&A, 442, 587

Vink, J. S., de Koter, A., \& Lamers, H. J. G. L. M. 2001, A\&A, 369, 574

Vink, J. S., Drew, J. E., Harries, T. J., \& Oudmaijer, R. D. 2002, MNRAS, 337, 356

Vink, J. S., Drew, J. E., Harries, T. J., Oudmaijer, R. D., \& Unruh, Y. 2005 , MNRAS, 359, 1049

Vreeswijk, P. M., Ellison, S. L., Ledoux, C., et al. 2004, A\&A, 419, 927

Wolf, C., \& Podsiadlowski, P. 2007, MNRAS, 375, 1049

Woosley, S. E. 1993, ApJ, 405, 273

Woosley, S. E., \& Heger, A. 2006, ApJ, 637, 914

Yoon, Y.-C., \& Langer, N. 2005, A\&A, 443, 643 